\title{
Timely Reminders from COVID-19 for Dementia Care
}

\author{
Chin Yee Cheong, ${ }^{1} M B B S, M R C P$, Philip LK Yap, ${ }^{1} M B B S, M R C P$
}

The scale of COVID-19 has been unprecedented in its global impact and percolation through all strata of society. The evolving pandemic inevitably magnifies the susceptibility of the frail and vulnerable, of which people with dementia (PWD) form a significant proportion. ${ }^{1}$ As has been seen in other populations such as nursing home residents, there can be significant health and socio-economic implications if the special needs of vulnerable populations are not adequately addressed. ${ }^{2}$

Cognitive deficits in PWD limit their ability to understand and retain information. Hence, it can be challenging for them to appreciate the need for hygiene measures or to don a mask, as well as physical distancing and restrictions on leaving their homes. Such lack of insight can result in behaviours that increase the likelihood of exposure to infections and indirectly put their families and caregivers at risk. Effective ways to impart bite-size information tailored to the capacities of PWD are necessary. These can include the display of posters with large fonts and pictorials in strategic locations, and communicating in clear, concise and simple language. The Singapore government has also kept the public abreast with the progress of the outbreak through regular public engagement and news broadcast. ${ }^{3}$ By tapping into implicit memory that remains largely intact in mild to moderate dementia, repetitions can be helpful, as can patience and kindness. ${ }^{4}$

If PWD do head out, it is best they are accompanied by their caregivers. When this is not possible, they can carry with them expedient means of identification. In this regard, the Alzheimer's Association of Singapore, in conjunction with the National Council of Social Service, has deployed the Safe Return Card for several years. ${ }^{5}$ In addition, In Case of Emergency, Dial (ICED) stickers can also be attached to the apparel of PWD. If possible, they should always be reachable through a handphone or GPS tracking device with their expressed consent. The recently launched 'Support for Persons living with dementia
Over the COVID-19 period' (SPOC-19) incorporates these measures, together with a memo carried by PWD that explains their condition. ${ }^{6}$

PWD generally do well when they keep to a structured daily routine with minimal changes. ${ }^{7}$ As a result of lockdown measures, these routines are disrupted and impact, in particular, those who go outdoors regularly. ${ }^{8}$ PWD may leave their place of residence and not comply with hygiene and safe distancing measures. Enforcement officers or the general public, unaware of the PWD's condition, may mistakenly accuse them of flouting regulations and enter into altercations that might trigger confusion and agitation. To reduce the need to go outdoors, caregivers can incorporate new activities that enable PWD to expend their energies and spend their time meaningfully. These activities can include daily exercises and engagement in telecognitive stimulation therapy or rehabilitation programmes, which could potentially have benefits comparable to conventional interventions. ${ }^{9}$

With the implementation of stay-home measures and suspension of day-care services, families of PWD who have been relying on such services may find themselves cut off from the support they have become dependent on. Should these families also find themselves unable to secure a stable income during the pandemic, the ordeal can be especially onerous. Moreover, as some low-income families may have limited awareness of the available avenues of assistance, it is necessary to be cognisant of their needs and consciously reach out to them. The support provided by the Chinese University of Hong Kong, which ranges from a national helpline to virtual dementia nurse service and family caregiver training, is a laudable example worth emulating. ${ }^{10}$ It is a platform for caregivers to seek ad hoc and continuous effective support that is backed by research. ${ }^{11}$

Should PWD become ill, caregivers may hesitate to send them to the hospital as it could mean that their loved ones are isolated and separated from them. The incidence of

\footnotetext{
${ }^{1}$ Department of Geriatric Medicine, Khoo Teck Puat Hospital, Singapore Address for Correspondence: Dr Cheong Chin Yee, Department of Geriatric Medicine, Khoo Teck Puat Hospital, 90 Yishun Central, Singapore 768828. Email: cheong.chin.yee@ktph.com.sg
} 
delirium in hospitalised older adults is as high as $56 \%$, especially in those with dementia. ${ }^{12}$ PWD may find it challenging to understand why they are confined to an unfamiliar place with restrictions on their freedom and disruption to their routines. Moreover, the inflammatory cytokines released from sepsis or COVID-19 could worsen delirium. ${ }^{13}$

Best practices for delirium assessment, mitigation and management should be adopted as far as possible without compromising priorities in infection control. For example, Yishun Health adopts the protocol, KNOW our VIPS NEEDS BEST ${ }^{3}$ (Table 1), which incorporates comprehensive geriatric assessment, person-centred care and collaborative delirium prevention care pathways, which can be adapted in light of pandemic measures. ${ }^{14,15}$ Even when no family members or caregivers are allowed to visit, leveraging on technology by means of video calls or telepresence robots enables caregivers and loved ones to render reassurance and comfort. ${ }^{16,17}$ Technology has more to offer. COVID-19 has led to a surge in demand for telemedicine as a means to ensure care continuity from the hospital to the community and even to patients' homes. As such, the role of telemedicine can be expanded to support patients cared for in nursing homes and their own homes where they typically do better unless they are gravely ill.

Table 1. The enhanced care protocol with the acronym KNOW our VIPS NEEDS BES ${ }^{3} \mathrm{~T}$

\begin{tabular}{|ll}
\hline V & Value (respect) \\
\hline I & Individual, Identity \\
\hline P & Perspective \\
\hline S & Social (relational) \\
\hline N & Normalise \\
\hline E & Engage \\
\hline E & Emancipate \\
\hline D & Dignify \\
\hline S & Simplify (slow) \\
\hline B & Bladder, Bowel, Brain \\
\hline E & Energy, Electrolytes, Environment \\
\hline S & Sight, Sound, Smile \\
\hline S & Sip, Stand, Sway \\
\hline S & Sleep, Skin, Strain \\
\hline
\end{tabular}

The high mortality rates of seniors stricken with COVID-19 inevitably highlight the importance of advance care planning even as many seniors remain phlegmatic about it. Interactions with PWD have taught us that some prefer not to deliberate over such issues while others see no need for it. Decisions on whether the benefits of hospitalisation outweigh the possible harms are particularly pertinent during the COVID-19 pandemic. The extent of treatment should also be thought through given the poor treatment outcomes in frail older people afflicted with COVD-19. ${ }^{18}$ For PWD who lack the mental capacity to make informed decisions, family members should arrive at a consensus in advance with informed and clear goals for their loved ones. ${ }^{19}$ If an advance care plan is not available, adopting a structured informed assent approach in the discussion of code status can be feasible, especially when active resuscitation is deemed futile. This framework enables clinicians to have high-quality conversations with families and uses patients' values to formulate an overall treatment goal. ${ }^{20}$

It is noteworthy that several of the recommendations proposed amid the present pandemic are not new and have at various times been advocated in the past. The initiative to build a dementia-friendly Singapore was conceived in 2016. ${ }^{21}$ It informs the public on how to recognise and assist PWD, and teaches practical ways to communicate and engage with them. The call for better awareness and support of caregivers has been constant over the years as has the need to improve hospital care for PWD, to minimise risks of enforced dependence and excess disability. Finally, making advance care plans in a disease like dementia that invariably renders one incapable of making informed decisions at some point is ever relevant and salient. COVID-19 has provided fresh impetus to take these recommendations to a renewed level of commitment so that PWD, their caregivers and the society as a whole can reap their benefits and leave no one behind.

\section{REFERENCES}

1. Onder G, Rezza G, Brusaferro S. Case-Fatality Rate and Characteristics of Patients Dying in Relation to COVID-19 in Italy. JAMA 2020; 323:1775-6

2. Penning MJ, Wu Z. Caregiver Stress and Mental Health: Impact of Caregiving Relationship and Gender. Gerontologist 2016;56:1102-13.

3. Ho CS, Chee CY, Ho RC. Mental Health Strategies to Combat the Psychological Impact of COVID-19 Beyond Paranoia and Panic. Ann Acad Med Singap 2020;49:155-60.

4. de Werd MME, Boelen D, Olde Rikkert MGM, et al. Errorless learning of everyday tasks in people with dementia. Clin Interv Aging 2013; $8: 1177-90$ 
5. Agelessonline. A safe return. 28 Jun 2012. Available at: https://www. agelessonline.net/a-safe-return/. Accessed on 6 May 2020.

6. The Straits Times. New memo to identify those with dementia who might unknowingly flout Covid-19 measures. 5 May 2020. Available at: https://www.straitstimes.com/singapore/new-memo-to-identifypersons-with-dementia-who-might-unknowingly-flout-covid-19-safe. Accessed on 6 May 2020.

7. Hodgson N, Safi AJ, Hodgson NA. Timing of Activities and their Effects on Circadian Rhythm in the Elderly with Dementia: A Literature Review. J Sleep Disord Ther 2014;3:176.

8. Business Insider. A running list of countries that are on lockdown because of the coronavirus pandemic. 24 September 2020. Available at: https://www.businessinsider.sg/countries-on-lockdown-coronavirusitaly-2020-3?r=US\&IR=T. Accessed on 30 October 2020

9. Cotelli M, Manenti R, Brambilla M, et al. Cognitive telerehabilitation in mild cognitive impairment, Alzheimer's disease and frontotemporal dementia: A systematic review. J Telemed Telecare 2019;25:67-79.

10. Jockey Club Post-diagnotic Support in Dementia Care Programme 2020. Available at: https://adcarer.com/eng/index.php?lang=en.Accessed on 6 May 2020

11. Kwok T, Au A, Wong B, et al. Effectiveness of online cognitive behavioral therapy on family caregivers of people with dementia. Clin Interv Aging 2014;9:631-6.

12. Fong TG, Tulebaev SR, Inouye SK. Delirium in elderly adults: Diagnosis, prevention and treatment. Nat Rev Neurol 2009;5:210-20.

13. Mehta P, McAuley DF, Brown M, et al. COVID-19: consider cytokine storm syndromes and immunosuppression. Lancet 2020;395:1033-4.
14. Cheong CY, Yap PLK. Staying true to the calling of geriatric medicine amid the waves of COVID-19. J Nutr Health Aging 2020;24:534-5.

15. LaHue SC, James TC, Newman JC, et al. Collaborative Delirium Prevention in the Age of COVID-19. J Am Geriatr Soc 2020;68:947-9.

16. TODAY. Robot to deliver meals, medication to Covid-19 patients at Alexandra Hospital to reduce exposure of healthcare workers. 5 March 2020. Available at: https://www.todayonline.com/singapore/robotdeliver-meals-medication-covid-19-patients-alexandra-hospital-reduceexposure. Accessed on 6 May 2020.

17. Van der Ploeg ES, Eppingstall B, O'Connor DW. Internet video chat (Skype) family conversations as a treatment of agitation in nursing home residents with dementia. Int Psychogeriatr 2016;28:697-8.

18. Alzheimer's Disease International. ADI offers advice and support during COVID-19. Available at: https://www.alz.co.uk/news/adi-offers-adviceand-support-during-covid-19. Accessed on 1 Jul 2020.

19. Tan LL, Ong PS, Ng LL, et al. Decision-Making in Dementia Care: a Qualitative Study of Chinese Family Caregivers in Singapore. Ann Acad Med Singap 2020;49:263-7.

20. Curtis JR, Kross EK, Stapleton RD. The Importance of Addressing Advance Care Planning and Decisions about Do-Not-Resuscitate Orders during Novel Coronavirus 2019 (COVID-19). JAMA 2020;323:1771-2.

21. The Straits Times. Two more dementia friendly towns soon. 23 February 2017. Available at: https://www.straitstimes.com/singapore/ two-more-dementia-friendly-towns-soon. Accessed on 6 May 2020. 